\title{
Evaluation of ultrasonography on carotid arteries and left ventricular function in patients with subclinical atherosclerosis
}

\author{
TAO ZHANG, HAIWEI YIN and HANWEN XUE
}

Department of Ultrasonography, Tengzhou Central People's Hospital, Tengzhou, Shandong 277599, P.R. China

Received November 23, 2018; Accepted April 12, 2019

DOI: $10.3892 / \mathrm{etm} .2019 .7517$

\begin{abstract}
Ultrasonography in the evaluation and diagnosis of carotid arteries and left ventricular function in patients with subclinical atherosclerosis (SA) was explored. In total, 152 patients with no obvious clinical symptoms of atherosclerosis confirmed by carotid ultrasonography in Tengzhou Central People's Hospital from September 2015 to March 2016 were enrolled as the experimental group, and further 45 patients with normal physical examination at the same time were collected as the control group. Patients in the experimental group were divided into three groups according to Framingham risk assessment: low, middle and high risk. The carotid arteries and left ventricular function of all patients were detected by Doppler ultrasound. There were differences in the systolic pressure, TG and TC between the control group and the experimental group $(\mathrm{P}<0.05)$. The expression levels of $\beta$, cIMT, Ep and PWV $\beta$ increased with the risk degree in the low, middle and high risk groups $(\mathrm{P}<0.05)$. The expression levels of LVEDV, LVESV, and LVEF in the experimental group were significantly decreased compared with those in the control group $(\mathrm{P}<0.05)$, while the expression levels of LAV, E/e, GLS and GCS were significantly increased compared with those in the control group $(\mathrm{P}<0.05)$. There was a difference of left ventricular function parameters in the low, medium and high risk groups $(\mathrm{P}<0.05)$. The expression levels of LVEDV, LVESV, LAV and E/e increased with the risk degree in the low, middle and high risk groups $(\mathrm{P}<0.05)$. The detection rate of plaque was lower in the low risk group than those in the middle and high risk group $(\mathrm{P}<0.05)$. By observing the parameters of carotid arteries and left ventricular function, it was found that ultrasonography has important clinical value in the early diagnosis of SA, and can be promoted in clinic.
\end{abstract}

Correspondence to: Dr Hanwen Xue, Department of Ultrasonography, Tengzhou Central People's Hospital, 181 Xingtan Road, Tengzhou, Shandong 277599, P.R. China

E-mail:we5q29@163.com

Key words: atherosclerosis, left ventricle, carotid ultrasound, cardiac ultrasound

\section{Introduction}

As the most important public health problem in the world, cardiovascular and cerebrovascular diseases were reported to have a death toll of 8.76 million in 2015 , ranking first among the 10 leading causes of death according to the World Health Organization (WHO) (1). The number of death in China is second only to malignant tumors (2). Atherosclerosis usually refers to the stenosis or obstruction of the lumen due to coronary atherosclerosis, resulting in local ischemia and hypoxia (3). Subclinical atherosclerosis (SA), a type of atherosclerosis, refers to the presence of atherosclerotic plaque in patients, without any clinical symptom (4). A study (5) showed that in recent years, the incidence of SA has decreased in developed countries, but increased in developing countries, which may be caused by the changes in dietary patterns as a result of economic development. Moreover, high fat diet and low cholesterol consumption play an important role in the pathogenesis of SA.

In the study of Weber et al (6), more than $50 \%$ of the patients with first cardiovascular and cerebrovascular disease did not have definite clinical symptoms. Therefore, early detection of cardiovascular function and intervention through clinical protocols in patients with SA can reduce the risk of cardiovascular diseases (7). With the continuous improvement of medical equipment, the diagnostic methods for atherosclerosis have been increasing, and the importance of ultrasonography in the diagnosis of atherosclerosis has been widely recognized in clinical practice (8). As a safe, rapid and noninvasive imaging tool, ultrasonography can directly reflect the structure and function of the heart and blood vessels in patients (9).

Therefore, the carotid arteries and left ventricular function of patients with SA were detected by ultrasonography in this study to provide references for diagnosis in clinic.

\section{Patients and methods}

Clinical data. Retrospective analysis was carried out of 152 patients with no obvious clinical symptoms of atherosclerosis as confirmed by carotid ultrasonography in Tengzhou Central People's Hospital (Tengzhou, China) from September 2015 to March 2016. These patients were enrolled as the experimental group, and 45 patients with normal liver, kidney, heart function, no stroke, myocardial infarction history examined in the above 
Table I. Comparison of clinical data between the two groups [n (\%)].

\begin{tabular}{|c|c|c|c|c|}
\hline Factor & Control group $(n=45)$ & Experimental group $(n=139)$ & $t / \chi^{2}$ value & P-value \\
\hline Sex & & & 0.083 & 0.773 \\
\hline Male & $27(60.00)$ & $80(57.55)$ & & \\
\hline Female & $18(40.00)$ & $59(42.25)$ & & \\
\hline Age (years) & & & 0.422 & 0.516 \\
\hline$\geq 60$ & $29(64.44)$ & $82(58.99)$ & & \\
\hline$<60$ & $16(35.56)$ & $57(41.01)$ & & \\
\hline Smoking history & & & 0.337 & 0.562 \\
\hline Yes & $15(33.33)$ & $40(28.78)$ & & \\
\hline No & $30(66.67)$ & $99(71.22)$ & & \\
\hline BMI $\left(\mathrm{kg} / \mathrm{m}^{2}\right)$ & $23.54 \pm 3.25$ & $23.84 \pm 4.25$ & 0.434 & 0.665 \\
\hline FPG (mmol/l) & $5.36 \pm 0.68$ & $5.29 \pm 0.70$ & 0.587 & 0.558 \\
\hline Systolic pressure (mmHg) & $129.50 \pm 15.84$ & $146.35 \pm 25.24$ & 4.213 & $<0.001$ \\
\hline Diastolic pressure (mmHg) & $76.84 \pm 7.54$ & $79.54 \pm 9.44$ & 1.746 & 0.083 \\
\hline $\mathrm{TG}(\mathrm{mmol} / \mathrm{l})$ & $1.18 \pm 0.52$ & $4.59 \pm 1.84$ & 12.254 & $<0.001$ \\
\hline $\mathrm{TC}(\mathrm{mmol} / \mathrm{l})$ & $3.25 \pm 1.05$ & $4.82 \pm 1.63$ & 6.061 & $<0.001$ \\
\hline $\mathrm{HDL}(\mathrm{mmol} / \mathrm{l})$ & $1.33 \pm 0.60$ & $1.35 \pm 0.62$ & 0.189 & 0.850 \\
\hline LDL (mmol/l) & $2.69 \pm 1.01$ & $2.48 \pm 0.84$ & 1.385 & 0.168 \\
\hline
\end{tabular}

BMI, body mass index; FPG, fasting plasma glucose; TG, triglycerides; TC, total cholesterol; HDL, high density lipoprotein; LDL, low density lipoprotein.

hospital at the same time were collected as the control group. Inclusion criteria were: patients with complete clinical data, no malignant tumor, in accordance with SA diagnostic criteria. Exclusion criteria were: patients with coronary atherosclerotic heart disease, heart valve disease, renal failure, immune deficiency disease, stroke and transient cerebral ischemia history. In total 139 cases were enrolled in this study according to the above criteria. This study was approved by the Medical Ethics Committee of Tengzhou Central People's Hospital. Patients who participated in this research had complete clinical data. Signed informed consents were obtained from the patients or the guardians.

Detection methods and data collection. MyLab90 color Doppler ultrasound (Yum Company, Italy) with Echo-tracking technology, digital image management system and LA523 probe was used at a frequency of 4-13 MHz. Philips iE33 color Doppler ultrasound with TomTec workstation (3D speckle tracing imaging technology) and S/X5-1 probe was used at a frequency of 2-5 MHz. Carotid arteries and left ventricular function in the two groups were measured. The parameters of common carotid artery function and left ventricular function in the two groups were collected. The serum was collected and the blood lipid of patients was detected by Hitachi 7600 automatic biochemical analyzer. Fasting plasma glucose (FPG), BMI index, blood pressure and plaque detection rate were analyzed.

Subgroups in the experimental group. According to the Framingham risk assessment, the incidence probability of coronary disease in the next 10 years of the 139 patients in the experimental group was analyzed (indicators included age, total cholesterol, high density lipoprotein, systolic pressure and smoking). In total, 46 patients, including 26 males and 20 females, were included in the low risk group (incidence $<10 \%$ ); 47 patients, including 25 males and 22 females, were included in the medium risk group $(>10 \%-<20 \%$ incidence); 46 patients, including 29 males and 17 females, were included in the high risk group (incidence $>20 \%$ ).

Statistical analysis. SPSS 20.0 software package was used for statistical analysis of the collected data. GraphPad Prism 7 software was used to draw figures. Countable data were expressed as rate (\%), analyzed by Chi-square test $\left(\chi^{2}\right)$. The measurement data are expressed as means $\pm \mathrm{SD}$. Comparison between the two groups was conducted by independent sample t-test and expressed by t-test. Rank data were analyzed by rank-sum test and expressed by Z. Multigroup comparison was conducted by single factor ANOVA, and post pairwise comparison was analyzed by LSD-t test and expressed by F. P $<0.05$ was considered to indicate a statistically significant difference.

\section{Results}

Analysis of clinical data of patients in the control group and the experimental group. The clinical data in the two groups showed that, in the control group, there were 27 males and 18 females, 29 patients were aged $>60$ years, and 15 patients smoked. BMI $\left(\mathrm{kg} / \mathrm{m}^{2}\right): 23.54 \pm 3.25$, FPG (mmol/l): 5.36 \pm 0.68 , systolic pressure $(\mathrm{mmHg}): 129.50 \pm 15.84$, diastolic pressure (mmHg): 76.84 7.54 , TG (mmol/l): $1.18 \pm 0.52$, TC (mmol/l): $3.25 \pm 1.05, \mathrm{HDL}(\mathrm{mmol} / \mathrm{l}): 1.33 \pm 0.60, \mathrm{LDL}(\mathrm{mmol} / \mathrm{l}): 2.69 \pm 1.01$. 
Table II. Comparison of carotid artery parameters between patients in the experimental and control groups.

\begin{tabular}{|c|c|c|c|c|}
\hline Index & Control group $(n=45)$ & Experimental group $(n=139)$ & $\mathrm{t}$ value & P-value \\
\hline$\beta$ value & $6.24 \pm 1.05$ & $10.90 \pm 4.56$ & 6.786 & $<0.001$ \\
\hline cIMT (mm) & $0.69 \pm 0.13$ & $0.96 \pm 0.19$ & 8.876 & $<0.001$ \\
\hline Ep $(\mathrm{kPa})$ & $78.64 \pm 20.18$ & $140.45 \pm 61.93$ & 6.573 & $<0.001$ \\
\hline $\mathrm{AI}(\%)$ & $19.88 \pm 8.45$ & $42.17 \pm 9.47$ & 14.075 & $<0.001$ \\
\hline $\mathrm{AC}\left(\mathrm{mm}^{2} / \mathrm{kPa}\right)$ & $0.84 \pm 0.22$ & $2.63 \pm 0.79$ & 14.987 & $<0.001$ \\
\hline $\mathrm{PWV} \beta(\mathrm{min} / \mathrm{sec})$ & $4.78 \pm 1.20$ & $7.45 \pm 1.50$ & 10.862 & $<0.001$ \\
\hline
\end{tabular}

$\beta$, vascular wall stiffness; cIMT, carotid intima-media thickness; Ep, vascular pressure-strain elastic modulus; AI, amplification index; AC, arterial vessel compliance; PWV $\beta$, single point pulse wave velocity.

Table III. Comparison of carotid artery parameters in the low, medium and high risk groups.

\begin{tabular}{|c|c|c|c|c|c|}
\hline Index & Low risk group $(n=46)$ & Medium risk group $(n=47)$ & High risk group $(n=46)$ & $\mathrm{F}$ value & P-value \\
\hline$\beta$ value & $8.84 \pm 2.54$ & $10.94 \pm 3.54^{\mathrm{a}}$ & $13.22 \pm 4.81^{\mathrm{a}, \mathrm{b}}$ & 15.735 & $<0.001$ \\
\hline cIMT (mm) & $0.84 \pm 0.18$ & $0.99 \pm 0.17^{\mathrm{a}}$ & $1.08 \pm 0.14^{\mathrm{a}, \mathrm{b}}$ & 25.070 & $<0.001$ \\
\hline $\mathrm{Ep}(\mathrm{kPa})$ & $120.84 \pm 41.55$ & $149.35 \pm 45.37^{\mathrm{a}}$ & $186.47 \pm 79.51^{\mathrm{a}, \mathrm{b}}$ & 14.800 & $<0.001$ \\
\hline $\mathrm{AI}(\%)$ & $42.44 \pm 15.28$ & $49.28 \pm 15.66$ & $46.10 \pm 15.75$ & 2.247 & 0.110 \\
\hline $\mathrm{AC}\left(\mathrm{mm}^{2} / \mathrm{kPa}\right)$ & $0.79 \pm 0.29$ & $0.77 \pm 0.22$ & $0.75 \pm 0.32$ & 0.236 & 0.790 \\
\hline $\mathrm{PWV} \beta(\mathrm{min} / \mathrm{sec})$ & $6.88 \pm 1.62$ & $7.35 \pm 1.25^{\mathrm{a}}$ & $8.29 \pm 1.71^{\mathrm{a}, \mathrm{b}}$ & 10.033 & $<0.001$ \\
\hline
\end{tabular}

$\beta$, vascular wall stiffness; cIMT, carotid intima-media thickness; Ep, vascular pressure-strain elastic modulus; AI, amplification index; AC, arterial vessel compliance; $\mathrm{PWV} \beta$, single point pulse wave velocity. ${ }^{\mathrm{a}} \mathrm{P}<0.05$, compared to low risk group; ${ }^{\mathrm{b}} \mathrm{P}<0.05$, compared to medium risk group.

In the experimental group, there were 80 males and 59 females, 82 patients were aged $>60$ years, and 40 patients smoked. BMI $\left(\mathrm{kg} / \mathrm{m}^{2}\right): 23.84 \pm 4.25$, FPG (mmol/1): 5.29 \pm 0.70 , systolic pressure $(\mathrm{mmHg}): 146.35 \pm 25.24$, diastolic pressure $(\mathrm{mmHg})$ :

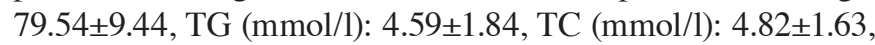
HDL (mmol/l): 1.35 \pm 0.62 , LDL (mmol/l): $2.48 \pm 0.84$. There was no significant difference in gender, age, smoking history, BMI, FPG, diastolic pressure, HDL and LDL between the two groups $(\mathrm{P}>0.05)$, while there were differences in the systolic pressure, TG and TC $(\mathrm{P}<0.05)$ (Table I).

Comparison of carotid artery parameters of patients. By comparing the carotid artery parameters of the two groups, it was found that the expression levels of $\beta$, cIMT, Ep, AI, AC and $\mathrm{PWV} \beta$ in the experimental group were significantly increased compared with those in the control group $(\mathrm{P}<0.05)$ (Table II). Comparison of arterial parameters in the low, middle and high risk groups according to the Framingham risk assessment found that there was no statistical difference in $\mathrm{AC}$ and $\mathrm{AI}$ among the three groups $(\mathrm{P}>0.05)$, but there was a statistical difference in other indexes $(\mathrm{P}<0.05)$. The expression levels of $\beta$, cIMT, Ep, PWV $\beta$ increased with the risk degree in the low, medium and high risk groups, and there were differences among the groups $(\mathrm{P}<0.05)$ (Table III).

Left ventricular function parameters of patients. Comparison of left ventricular function parameters between the two groups found that the expression levels of LVEDV, LVESV, and
LVEF in the experimental group were significantly decreased compared with those in the control group $(\mathrm{P}<0.05)$, while the expression levels of LAV, E/e, GLS and GCS were significantly increased compared with those in the control group $(\mathrm{P}<0.05)$ (Table IV). Comparison of left ventricular function parameters in the low, medium and high risk groups according to Framingham risk assessment found that there were significant differences among the three groups $(\mathrm{P}<0.05)$. The expression levels of LVEDV, LVESV, LAV and E/e increased with the risk degree in the three groups $(\mathrm{P}<0.05)$. There was no significant difference in GLS expression between low and middle risk groups $(\mathrm{P}>0.05)$, while the expression was significantly increased compared with that in the high risk group $(\mathrm{P}<0.05)$, and there was no significant difference in LVEF and GCS among the three groups $(\mathrm{P}>0.05)$ (Table V).

Plaque detection in the experimental group. Analysis of plaque detection rate in the experimental group found that the detection rate of plaque was $21.74 \%(n=10)$ in the low-risk group, $48.94 \%(\mathrm{n}=23)$ in the medium risk group and $58.70 \%$ $(n=27)$ in the high-risk group. There were significant differences in plaque detection rate among the three groups $(\mathrm{P}<0.05)$ (Table VI and Fig. 1).

\section{Discussion}

Cardiovascular and cerebrovascular diseases are the most prevalent clinical diseases in the world, and coronary heart 
Table IV. Comparison of left ventricular function parameters of patients in the experimental and control groups.

\begin{tabular}{|c|c|c|c|c|}
\hline Index & Control group $(n=45)$ & Experimental group $(n=139)$ & $\mathrm{t}$ value & P-value \\
\hline LVEDV (ml) & $83.41 \pm 18.24$ & $63.10 \pm 15.61$ & 7.272 & $<0.001$ \\
\hline LVESV (ml) & $35.84 \pm 9.24$ & $22.25 \pm 7.56$ & 9.906 & $<0.001$ \\
\hline $\operatorname{LVEF}(\%)$ & $70.54 \pm 16.38$ & $63.84 \pm 5.81$ & 4.107 & $<0.001$ \\
\hline LAV (ml) & $30.84 \pm 8.84$ & $43.69 \pm 14.06$ & 5.768 & $<0.001$ \\
\hline $\mathrm{E} / \mathrm{e}$ & $6.84 \pm 3.27$ & $11.68 \pm 3.35$ & 8.472 & $<0.001$ \\
\hline GLS $(\%)$ & $10.12 \pm 4.25$ & $20.57 \pm 3.59$ & 16.204 & $<0.001$ \\
\hline GCS $(\%)$ & $9.15 \pm 2.22$ & $31.92 \pm 5.67$ & 26.255 & $<0.001$ \\
\hline
\end{tabular}

LVEDV, left ventricular end-diastolic volume; LVESV, left ventricular end-systolic volume; LVEF, left ventricular ejection fraction; LAV, left atrium volume; E/e, ratio of mitral annular early diastolic velocity/mitral orifice early diastolic velocity; GLS, global longitudinal strain; GCS, global circumferential strain.

Table V. Comparison of left ventricular function parameters in the low, middle and high risk groups.

\begin{tabular}{|c|c|c|c|c|c|}
\hline Index & Low risk group $(n=46)$ & Medium risk group $(n=47)$ & High risk group $(n=46)$ & F value & P-value \\
\hline LVEDV (ml) & $56.47 \pm 10.84$ & $63.84 \pm 11.36^{\mathrm{a}}$ & $71.35 \pm 16.88^{\mathrm{a}, \mathrm{b}}$ & 14.402 & $<0.001$ \\
\hline LVESV (ml) & $19.35 \pm 5.11$ & $22.39 \pm 6.88^{\mathrm{a}}$ & $26.94 \pm 6.58^{\mathrm{a}, \mathrm{b}}$ & 17.225 & $<0.001$ \\
\hline $\operatorname{LVEF}(\%)$ & $63.87 \pm 5.89$ & $64.27 \pm 5.34$ & $62.25 \pm 4.39$ & 1.926 & 0.150 \\
\hline LAV (ml) & $35.04 \pm 8.05$ & $47.33 \pm 10.58^{\mathrm{a}}$ & $55.37 \pm 15.82^{\mathrm{a}, \mathrm{b}}$ & 33.940 & $<0.001$ \\
\hline $\mathrm{E} / \mathrm{e}$ & $10.52 \pm 2.67$ & $12.84 \pm 3.89^{\mathrm{a}}$ & $14.68 \pm 4.42^{\mathrm{a}, \mathrm{b}}$ & 14.339 & $<0.001$ \\
\hline GLS (\%) & $20.95 \pm 3.81$ & $20.17 \pm 3.80$ & $18.66 \pm 3.17^{\mathrm{a}, \mathrm{b}}$ & 4.794 & 0.010 \\
\hline GCS $(\%)$ & $34.25 \pm 5.29$ & $33.02 \pm 5.10$ & $31.95 \pm 6.17$ & 1.988 & 0.141 \\
\hline
\end{tabular}

LVEDV, left ventricular end-diastolic volume; LVESV, left ventricular end-systolic volume; LVEF, left ventricular ejection fraction; LAV, left atrium volume; E/e, ratio of mitral annular early diastolic velocity/mitral orifice early diastolic velocity; GLS, global longitudinal strain; GCS, global circumferential strain. ${ }^{a} \mathrm{P}<0.05$, compared to low risk group; ${ }^{b} \mathrm{P}<0.05$, compared to medium risk group.

disease is the most common disease (10). Atherosclerosis usually refers to the stenosis or obstruction of the lumen due to coronary atherosclerosis, resulting in local ischemia and hypoxia. A survey shows (11) that patients with coronary heart disease are getting younger as a result of improved living standards and changes in dietary habits. With the increasing incidence of coronary heart disease, it is receiving more attention. As a chronic disease process, atherosclerosis can be divided into pre-clinical stage and end-stage. End-stage patients mainly have coronary heart disease, myocardial infarction or ischemic stroke. Once patients enter the end-stage, they are prone to death. Patients who are rescued in time have a high disability rate, which seriously affects the life quality of patients. Patients in the pre-clinical stage, also known as SA, can have the presence of atherosclerosis through the corresponding examination without any corresponding clinical symptoms (12-14). A study has shown (15) that early intervention in patients at SA stage can delay the course of disease and reduce the occurrence of adverse reactions.

At present, Framingham risk assessment is commonly used to evaluate the incidence of coronary heart disease in patients, which calculates the incidence rate of coronary heart disease in the next 10 years according to the cholesterol level and non-cholesterol factors of the patients (16). Although the assessment evaluates patients through a variety of factors, it can not effectively diagnose and assess SA patients. Ultrasonography has been widely used in clinical imaging for many years and has been widely recognized by clinicians. It is non-invasive, safe, rapid and accurate, and has a high accuracy in the diagnosis of cardiovascular disease (17). Carotid arteries and left ventricular function between healthy subjects and patients with SA were compared and the carotid arteries and left ventricular function in patients with SA with different risk degrees were further analyzed in this study to provide a reference for clinical diagnosis.

Patients in the experimental group and the control group were examined by ultrasonography. The indexes of $\beta$, cIMT, Ep, AI, AC, PWV $\beta$ have been reported $(18,19)$ to be important for the early lesion of arterial wall, and were detected by Echo-tracking technique in this study. After analysis, it was found that the above indexes in the experimental group were significantly higher than those in the control group, and the stratification analysis of the experimental group showed that the expression of $\beta$, cIMT, Ep and PWV $\beta$ increased with the risk degree in the low, middle and high risk groups, indicating that with the increase of risk degree, the blood vessel wall gradually hardened, carotid intima thickness 
Table VI. Plaque detection in the experimental group [n (\%)].

\begin{tabular}{|c|c|c|c|c|}
\hline Group & Patients with plaques & Patients without plaques & $\mathrm{Z}$ value & P-value \\
\hline Low risk group $(n=46)$ & $10(21.74)$ & $36(78.26)$ & -3.565 & $<0.001$ \\
\hline Medium risk group $(n=47)$ & $23(48.94)$ & $24(51.06)$ & & \\
\hline High risk group $(n=46)$ & $27(58.70)$ & $19(41.30)$ & & \\
\hline
\end{tabular}

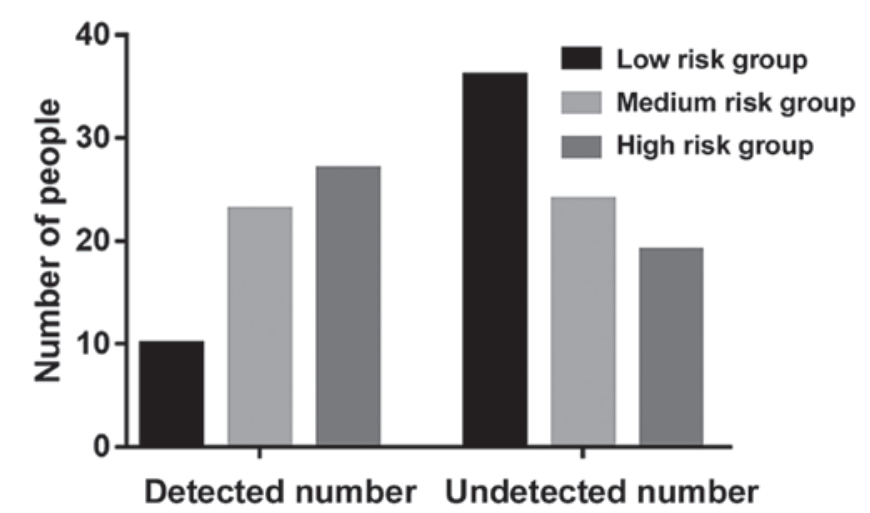

Figure 1. Plaque detection rate in the low, medium and high risk groups. The detection rate of plaque was $21.74 \%(n=10)$ in the low-risk group, $48.94 \%$ $(n=23)$ in the medium risk group and $58.70 \%(n=27)$ in the high-risk group. There were significant differences in plaque detection rate between the three groups $(\mathrm{P}<0.05)$.

increased, vascular elasticity gradually weakened, pulse wave velocity increased, which increased the risk of adverse cardiovascular events. It was assumed that this was mainly due to the stimulation of the vascular intima under the influence of various risk factors, thereby destroying the vascular elastic fibers, causing smooth muscle proliferation, vascular remodeling and inflammation, ultimately, leading to the above symptoms (20-22). Then the left ventricular function of the patients in the experimental group and the control group was measured by 3D speckle tracing imaging technology that is a 3D spatial quantitative measurement of myocardial strain and strain rate, and can reflect the early contraction of myocardial fiber in patients $(23,24)$. The results showed that the LVEDV, LVESV and LVEF in the experimental group were significantly lower than those in the control group, while the LAV, E/e, GLS and GCS in the experimental group were significantly higher than those in the control group. Moreover, further analysis found that LVEDV, LVESV and E/e increased with the risk of the disease, while LVEF was the opposite. This was because the increase of $\beta$ reduced the reserve of vascular elasticity in the patient, and further caused the increase of systolic pressure and the decrease of diastolic pressure, therefore, causing myocardial ischemia in patients. A study by Agoşton-Coldea et al (25) showed that atherosclerosis occlusion in lower extremities is closely related to carotid stiffness and left ventricular diastolic function. The results of our study showed that there was no difference in the incidence of LVEF and GCS between the groups, but the incidence of GLS in the low and medium risk groups was significantly higher than that in the high risk group, and there was no significant difference between low and medium risk groups. This suggests that the decrease of vascular elasticity does not affect LVEF and GCS, and it is only when the patient reaches high risk that the left ventricular GLS endocardial blood perfusion is decreased. At the end of the study, the carotid plaque in the experimental group was examined, and it was found that there were significant differences between the three groups, and with the increase of the degree of the disease, the number of detected carotid plaques increased significantly.

This study preliminarily proved the difference between the carotid artery parameters and left ventricular function in healthy subjects and SA patients, and further analyzed their relationship in patients with different risk degrees. However, there are still some limitations in this study. There may be some bias in the data in this study as a retrospective analysis. Follow-up analysis was not conducted, the specific adverse reactions are not clear. Therefore, clinical randomized controlled trials and follow-up studies are needed.

In conclusion, by observing the parameters of carotid arteries and left ventricular function, it was found that ultrasonography has important clinical value in the early diagnosis of $\mathrm{SA}$, and can be widely promoted in clinic.

\section{Acknowledgements}

Not applicable.

\section{Funding}

No funding was received.

\section{Availability of data and materials}

The datasets used and/or analyzed during the current study are available from the corresponding author on reasonable request.

\section{Authors' contributions}

TZ wrote the manuscript and analyzed the clinical data of patients. HY compared carotid artery parameters and left ventricular function parameters. HX was responsible for plaque detection. All authors read and approved the final manuscript.

\section{Ethics approval and consent to participate}

This study was approved by the Ethics Committee of Tengzhou Central People's Hospital (Tengzhou, China). Patients who participated in this research had complete clinical data. Signed informed consents were obtained from the patients or the guardians. 


\section{Patient consent for publication}

Not applicable.

\section{Competing interests}

The authors declare that they have no competing interests.

\section{References}

1. Dal-Ré R: Early phase drugs and biologicals clinical trials on worldwide leading causes of death: A descriptive analysis. Eur J Clin Pharmacol 67: 563-571, 2011.

2. Du H, Li L, Bennett D, Guo Y, Key TJ, Bian Z, Sherliker P, Gao H, Chen Y, Yang L, et al; China Kadoorie Biobank Study: Fresh fruit consumption and major cardiovascular disease in China. N Engl J Med 374: 1332-1343, 2016.

3. Al-Sharea A, Murphy AJ, Huggins LA, Hu Y, Goldberg IJ and Nagareddy PR: SGLT2 inhibition reduces atherosclerosis by enhancing lipoprotein clearance in $\mathrm{Ldlr}^{-/-}$type 1 diabetic mice. Atherosclerosis 271: 166-176, 2018.

4. Ambrosino P, Lupoli R, Di Minno A, Tasso M, Peluso R and Di Minno MN: Subclinical atherosclerosis in patients with rheumatoid arthritis. A meta-analysis of literature studies. Thromb Haemost 113: 916-930, 2015.

5. Benjamin EJ, Blaha MJ, Chiuve SE, Cushman M, Das SR, Deo R, de Ferranti SD, Floyd J, Fornage M, Gillespie C, et al; American Heart Association Statistics Committee and Stroke Statistics Subcommittee: Heart Disease and Stroke Statistics-2017 Update: A report from the American Heart Association. Circulation 135 e146-e603, 2017.

6. Weber LA, Cheezum MK, Reese JM, Lane AB, Haley RD Lutz MW and Villines TC: Cardiovascular imaging for the primary prevention of atherosclerotic cardiovascular disease events. Curr Cardiovasc Imaging Rep 8: 36, 2015.

7. de Almeida-Pititto B, Ribeiro-Filho FF, Bittencourt MS, Lotufo PA, Bensenor I and Ferreira SR: Usefulness of circulating E-selectin to early detection of the atherosclerotic process in the Brazilian Longitudinal Study of Adult Health (ELSA-Brasil). Diabetol Metab Syndr 8: 19, 2016.

8. Huang R, Abdelmoneim SS, Ball CA, Nhola LF, Farrell AM, Feinstein S and Mulvagh SL: Detection of carotid atherosclerotic plaque neovascularization using contrast enhanced ultrasound: A systematic review and meta-analysis of diagnostic accuracy studies. J Am Soc Echocardiogr 29: 491-502, 2016.

9. ten Kate GL, van Dijk AC, van den Oord SC, Hussain B, Verhagen HJ, Sijbrands EJ, van der Steen AF, van der Lugt A and Schinkel AF: Usefulness of contrast-enhanced ultrasound for detection of carotid plaque ulceration in patients with symptomatic carotid atherosclerosis. Am J Cardiol 112: 292-298, 2013.

10. Saleheen D, Scott R, Javad S, Zhao W, Rodrigues A, Picataggi A Lukmanova D, Mucksavage ML, Luben R, Billheimer J, et al: Association of HDL cholesterol efflux capacity with incident coronary heart disease events: A prospective case-control study. Lancet Diabetes Endocrinol 3: 507-513, 2015.

11. Wilmot KA, O'Flaherty M, Capewell S, Ford ES and Vaccarino V: Coronary heart disease mortality declines in the United States from 1979 through 2011: Evidence for stagnation in young adults, especially women. Circulation 132: 997-1002, 2015.
12. Poredoš $\mathrm{P}$ and Ježovnik MK: Markers of preclinical atherosclerosis and their clinical relevance. Vasa 44: 247-256, 2015.

13. McClelland RL, Jorgensen NW, Budoff M, Blaha MJ, Post WS Kronmal RA, Bild DE, Shea S, Liu K, Watson KE, et al: 10-Year coronary heart disease risk prediction using coronary artery calcium and traditional risk factors: Derivation in the MESA (Multi-Ethnic Study of Atherosclerosis) with validation in the HNR (Heinz Nixdorf Recall) Study and the DHS (Dallas Heart Study). J Am Coll Cardiol 66: 1643-1653, 2015.

14. Matsushita K, Sang Y, Ballew SH, Shlipak M, Katz R, Rosas SE, Peralta CA, Woodward M, Kramer HJ, Jacobs DR, et al: Subclinical atherosclerosis measures for cardiovascular prediction in CKD. J Am Soc Nephrol 26: 439-447, 2015.

15. Gourgari E, Dabelea D and Rother K: Modifiable risk factors for cardiovascular disease in children with type 1 diabetes: can early intervention prevent future cardiovascular events? Curr Diab Rep 17: 134, 2017.

16. Sansone R, Rodriguez-Mateos A, Heuel J, Falk D, Schuler D, Wagstaff R, Kuhnle GG, Spencer JP, Schroeter H, Merx MW, et al; Flaviola Consortium, European Union 7th Framework Program: Cocoa flavanol intake improves endothelial function and Framingham Risk Score in healthy men and women: A randomised, controlled, double-masked trial: the Flaviola Health Study. Br J Nutr 114: 1246-1255, 2015.

17. Rosengarten B and Kaps M: A simultaneous EEG and transcranial Doppler technique to investigate the neurovascular coupling in the human visual cortex. Cerebrovasc Dis 29: 211-216, 2010.

18. Yang S, Wang DZ, Zhang HX, He W and Chen BX: Echo-tracking technology assessment of carotid artery stiffness in patients with coronary slow flow. Ultrasound Med Biol 41: 72-76, 2015.

19. Zhang P, Guo R, Li Z, Xiao D, Ma L, Huang P and Wang C: Effect of smoking on common carotid artery wall elasticity evaluated by echo tracking technique. Ultrasound Med Biol 40: 643-649, 2014

20. Lee SJ and Park SH: Arterial ageing. Korean Circ J 43: 73-79, 2013.

21. Zhang P, Guo R, Xiao D, Chu S, Gong L, Zhang C, Jing B and Li M: Influence of smoking cessation on carotid artery wall elasticity evaluated by echo-tracking. J Clin Ultrasound 40: 352-356, 2012.

22. Nahrendorf M, Jaffer FA, Kelly KA, Sosnovik DE, Aikawa E, Libby $\mathrm{P}$ and Weissleder R: Noninvasive vascular cell adhesion molecule-1 imaging identifies inflammatory activation of cells in atherosclerosis. Circulation 114: 1504-1511, 2006.

23. Ling Y, Wan Q, Chen Q and Zhu W: Assessment of subtle cardiac dysfunction in patients with frequent premature ventricular complexes by real-time three-dimensional speckle tracking echocardiography. Clin Cardiol 40: 554-558, 2017.

24. Huang X, Kang X, Xue J, Kang C, Lv H and Li Z: Evaluation of carotid artery elasticity changes in patients with cerebral small vessel disease. Int J Clin Exp Med 8: 18825-18830, 2015.

25. Agoşton-Coldea L, Mocan T and Bobar C: Arterial stiffness and left ventricular diastolic function in the patients with hypertension. Rom J Intern Med 46: 313-321, 2008.

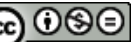

This work is licensed under a Creative Commons Attribution-NonCommercial-NoDerivatives 4.0 International (CC BY-NC-ND 4.0) License. 